\title{
Quasiparticle breakdown in a quantum spin liquid
}

\author{
Matthew B. Stone ${ }^{1}$, Igor A. Zaliznyak ${ }^{2}$, Tao Hong ${ }^{3}$, Collin L. Broholm ${ }^{3,4} \&$ Daniel H. Reich ${ }^{3}$
}

Much of modern condensed matter physics is understood in terms of elementary excitations, or quasiparticles-fundamental quanta of energy and momentum ${ }^{1,2}$. Various strongly interacting atomic systems are successfully treated as a collection of quasiparticles with weak or no interactions. However, there are interesting limitations to this description: in some systems the very existence of quasiparticles cannot be taken for granted. Like unstable elementary particles, quasiparticles cannot survive beyond a threshold where certain decay channels become allowed by conservation laws; their spectrum terminates at this threshold. Such quasiparticle breakdown was first predicted for an exotic state of matter-super-fluid ${ }^{4} \mathrm{He}$ at temperatures close to absolute zero, a quantum Bose liquid where zero-point atomic motion precludes crystallization $^{1-4}$. Here we show, using neutron scattering, that quasiparticle breakdown can also occur in a quantum magnet and, by implication, in other systems with Bose quasiparticles. We have measured spin excitations in a two-dimensional quantum magnet, piperazinium hexachlorodicuprate $(\mathrm{PHCC})^{5}$, in which spin-1/2 copper ions form a non-magnetic quantum spin liquid, and find remarkable similarities with excitations in superfluid ${ }^{4} \mathrm{He}$. We observe a threshold momentum beyond which the quasiparticle peak merges with the two-quasiparticle continuum. It then acquires a finite energy width and becomes indistinguishable from a leading-edge singularity, so that excited states are no longer quasiparticles but occupy a wide band of energy. Our findings have important ramifications for understanding excitations with gapped spectra in many condensed matter systems, ranging from band insulators to high-transition-temperature superconductors ${ }^{6}$.

Although of all the elements only liquid helium fails to crystallize at temperature $T=0$, quantum liquids are quite common in condensed matter. Metals host electron Fermi liquids, and superconductors contain Bose liquids of Cooper pairs. Trapped ultracold atoms can also form quantum liquids, and some remarkable new examples were recently identified in magnetic crystals ${ }^{5,7-10}$. The organometallic material PHCC is an excellent physical realization of a quantum spin liquid (QSL) in a two-dimensional (2D) Heisenberg antiferromagnet (HAFM). Its $\mathrm{Cu}^{2+}$ spins are coupled through a complex network of orbital overlaps, and form an array of slightly skewed anisotropic spin-1/2 ladders ${ }^{10}$ in the crystalline $a-c$ plane with highly frustrated super-exchange interactions ${ }^{5}$. The spin excitations in PHCC have a spectral gap $\Delta_{\mathrm{s}} \approx 1 \mathrm{meV}$ and nearly isotropic $2 \mathrm{D}$ dispersion in the $(h 0 l)$ plane with a bandwidth slightly larger than $\Delta_{\text {s. }}$. In the absence of a magnetic field, only the short-range dynamic spin correlations typical of a liquid exist: the spin gap precludes longrange magnetic order down to $T=0$. Here we explore magnetic excitations in PHCC via inelastic neutron scattering and compare the results with similar measurements in the quantum fluid ${ }^{4} \mathrm{He}$, emphasizing the effects where quasiparticle dispersion reaches the threshold for two-particle decay and interferes destructively with the continuum.
The properties of superfluid ${ }^{4} \mathrm{He}$ (ref. 4) can be explained by considering Bose quasiparticles with a finite-energy minimum (an energy gap) in their spectrum ${ }^{1,2}$. However, in a Bose quantum liquid, a spectral gap can produce an energy-momentum threshold where the quasiparticle description breaks down ${ }^{1-3}$. Beyond this threshold, single-particle states are no longer approximate eigenstates of the hamiltonian and the quasiparticle spectrum terminates. Neutron scattering experiments in ${ }^{4} \mathrm{He}$ indicate that the spectrum of quasiparticles (quanta of longitudinal sound waves also called phonons) ends when the phonon is able to decay into two 'rotons ${ }^{11-15}$. These rotons are phonons with roughly quadratic dispersion that occur near the dispersion minimum, which is at energy $\Delta \approx 0.74 \mathrm{meV}$ and wavevector $Q \approx 2 \AA^{-1}$ (compare Fig. 1, main panel). Spontaneous decays provide the only mechanism that destroys quasiparticles in ${ }^{4} \mathrm{He}$ at $T=0$. However, owing to the high density of two-roton states, this decay path is so effective that instead of acquiring a finite lifetime, the quasiparticles simply cease to exist. Specifically, the single-particle pole is absent in the Green's function of ${ }^{4} \mathrm{He}$ atoms for $Q>Q_{c}$ (where $Q_{c}$ is a threshold wavevector), so that the quasiparticle spectrum does not continue beyond the threshold ${ }^{1-3}$.

The excitation spectrum of superfluid ${ }^{4} \mathrm{He}$ as probed by neutron scattering is shown in Fig. 1, main panel. One can see the roton minimum in the dispersion and the spectrum termination point at $Q_{c} \approx 2.6 \AA^{-1}$. Near $Q_{c}$ the phonon hybridizes with two-roton excitations, its dispersion flattens, and spectral weight is transferred

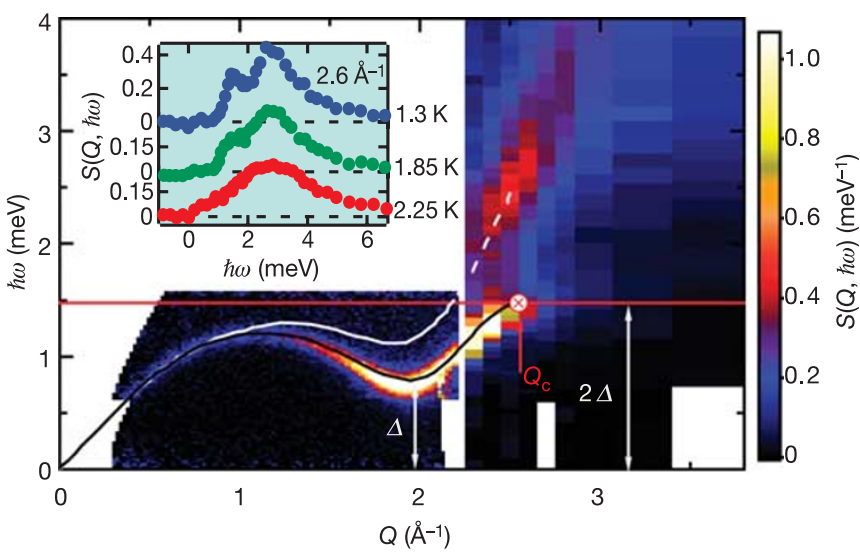

Figure 1 | Liquid helium excitation spectrum $S(Q, \hbar \omega)$ from inelastic neutron scattering measurements. Main panel, excitation spectrum in ${ }^{4} \mathrm{He}$ for $1.5 \leq T \leq 1.8 \mathrm{~K}$. Data for wavevector $Q \geq 2.3 \AA^{-1}$ are reproduced from ref. 13, data at smaller $Q$ are from C.L.B. and S.-H. Lee, unpublished results. Solid black line, dispersion from ref. 13; red circle with cross, spectrum termination point at $Q=Q_{\mathrm{c}}$ and $\hbar \omega=2 \Delta$. White line, Feynman-Cohen bare dispersion in absence of decays ${ }^{17}$; horizontal red line at $\hbar \omega=2 \Delta$, onset of two-roton states for $\hbar \omega \geq 2 \Delta$. Inset, excitations near termination point, at $Q=2.6 \AA^{-1} \approx Q_{c}$, for several temperatures ${ }^{13}$.

${ }^{1}$ Condensed Matter Sciences Division, Oak Ridge National Laboratory, Oak Ridge, Tennessee 37831, USA. ${ }^{2}$ Condensed Matter Physics and Material Science Department, Brookhaven National Laboratory, Upton, New York 11973, USA. ${ }^{3}$ Department of Physics and Astronomy, The Johns Hopkins University, Baltimore, Maryland 21218, USA. ${ }^{4}$ National Institute of Standards and Technology, Gaithersburg, Maryland 20899, USA 
to the multiparticle continuum ${ }^{13,15}$. While a smeared maximum occurs at the leading edge of the continuum for $Q>Q_{c}$ and appears to continue the quasiparticle dispersion relation, it is instead ascribed to a two-roton bound state (resonance) resulting from roton-roton interactions ${ }^{15,16}$. Decays modify the 'bare' Feynman-Cohen quasiparticle dispersion in ${ }^{4} \mathrm{He}$ (white line in Fig. 1, main panel) ${ }^{17}$. Instead of terminating where it reaches the energy $2 \Delta$, the quasiparticle spectrum is suppressed to lower energies at $Q \leq Q_{c}$, approaching the threshold energy $\hbar \omega=2 \Delta$ horizontally $^{3}$ (black line in Fig. 1, main panel).

The generality of the physics underlying quasiparticle breakdown in ${ }^{4} \mathrm{He}$ suggests that similar effects may occur in other quantum liquids. The quasiparticle instability in ${ }^{4} \mathrm{He}$ relies on the isotropic nature of the fluid: since the spectrum only depends on $|Q|$, the roton minimum produces a strong singularity in the density of states (DOS). For QSLs on a crystalline lattice, the DOS available for quasiparticle decays is enhanced by the absence of dispersion in certain directions that occurs in low-dimensional systems $(D<3)$ and in systems with competing interactions (frustration). Quasiparticle breakdown effects should thus be strongest in one-dimensional (1D) QSLs, such as spin-1 chains with a spectral gap ${ }^{18}$. Though the

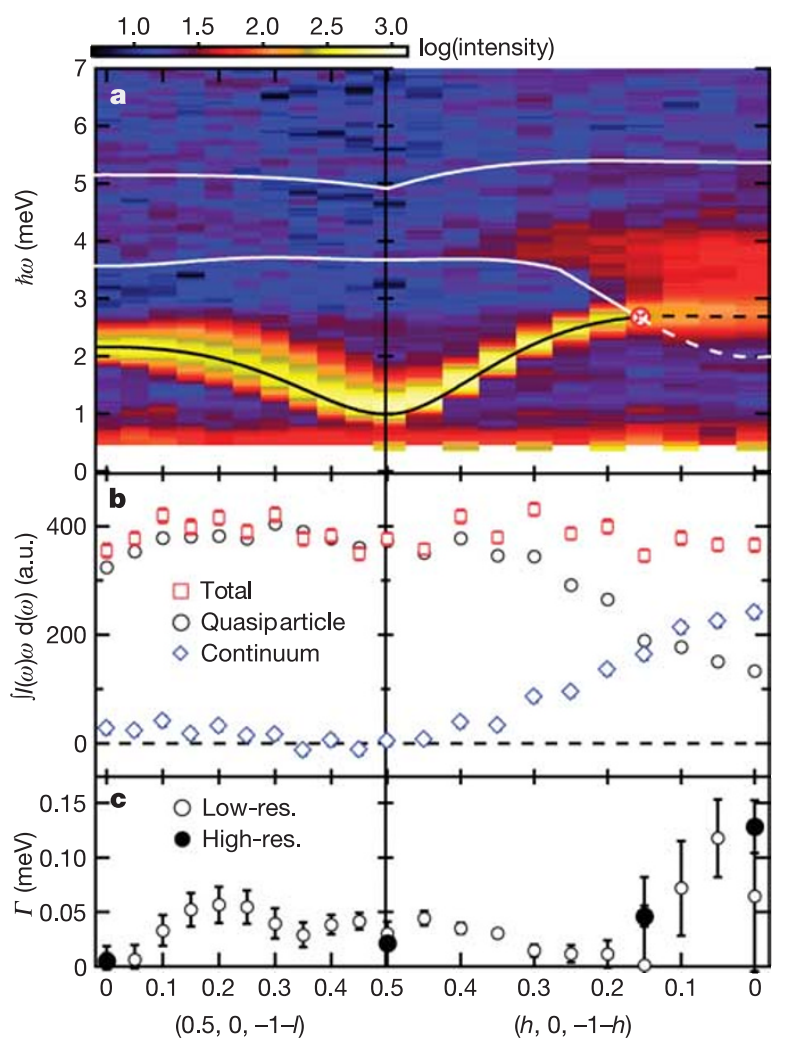

Figure 2 Magnetic excitation spectrum at $T=1.4 \mathrm{~K}$ in PHCC. a, Background-corrected intensity along the $(1 / 2,0,-1-l)$ and $(h, 0$,

$-1-h)$ directions. A $\delta \hbar \omega=0.25 \mathrm{meV}$ running average was applied to each constant wavevector scan, retaining the actual point density of the acquired data. Black line, previously determined single-magnon dispersion ${ }^{5}$. White lines, bounds of two-magnon continuum calculated from this dispersion. Red circle with cross, the point where the single-particle dispersion relation intersects the lower bound of the two-particle continuum. b. First frequency moment of measured scattering intensity integrated over different energy ranges. Red squares (total), $0.8 \leq \hbar \omega \leq 5.5 \mathrm{meV}$; black circles (quasiparticle), $0.8 \leq \hbar \omega \leq 3 \mathrm{meV}$; blue diamonds (continuum), $3 \leq \hbar \omega \leq 5.5 \mathrm{meV}$. c, Resolution-corrected half-width at half-maximum (HWHM) of the lower energy peak throughout the range of wavevector transfer for high resolution (solid points) and low resolution (open points) data. Error bars illustrate systematic error corresponding to $10 \%$ uncertainty in the neutron beam collimation used for resolution correction. term has not been used in this context, numerical work suggests that spectrum termination does occur in spin-1 HAFM spin chains ${ }^{19,20}$. Its observation through neutron scattering, however, is hindered by small scattering cross-sections at the appropriate wavevectors. In the spin-1 chain system $\mathrm{Ni}\left(\mathrm{C}_{2} \mathrm{H}_{8} \mathrm{~N}_{2}\right)_{2} \mathrm{NO}_{2} \mathrm{ClO}_{4}$ (NENP), scattering becomes undetectable when the single-particle excitation meets the non-interacting two-particle continuum ${ }^{21}$, owing to either decays or a vanishing structure factor. While transformation of magnetic excitations from well-defined quasiparticles to a continuum was observed in the quasi-1D spin-1 HAFM $\mathrm{CsNiCl}_{3}$, it is only seen as an onset of damping beyond a certain momentum threshold, well before the dispersion crosses the lower bound of the projected noninteracting two-particle continuum ${ }^{22}$, which may be a result of interchain interactions.

In contrast to the HAFM spin-1 chain, the structure factor of PHCC is favourable for probing the interaction of magnon quasiparticles with their two-particle continuum. Its effects, however, could be less pronounced because the 2D DOS singularities are weaker. Prior measurements examined magnetic excitations in PHCC below $\sim 3 \mathrm{meV}$ (ref. 5). Here we present data for energies $\hbar \omega \leq 7 \mathrm{meV}$ and for wavevectors along the $(1 / 2,0, l)$ and $(h, 0$, $-1-h)$ directions, elucidating both single- and multiparticle excitations in this 2D QSL. Data shown in Fig. 2a and selected scans shown in Fig. 3 demonstrate clear similarities to the spectrum of superfluid ${ }^{4} \mathrm{He}$. The one-magnon dispersion reaches the lower boundary of the two-magnon continuum, $\hbar \omega_{2 \mathrm{~m}}(\mathbf{Q})=$ $\min _{\mathrm{q}}\{\hbar \omega(\mathbf{q})+\hbar \omega(\mathbf{Q}-\mathbf{q})\}$, for $\mathbf{Q}_{\mathrm{c}}=\left(h_{\mathrm{c}}, 0,-1-h_{\mathrm{c}}\right)$ with

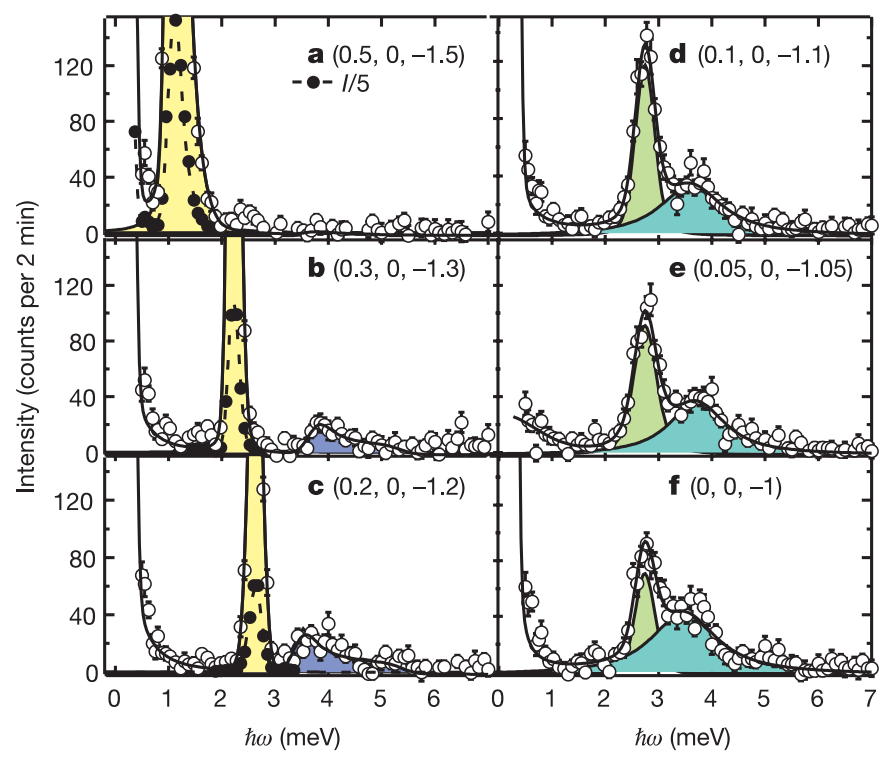

Figure 3 Individual constant-wavevector scans of PHCC along the ( $h, 0$, $-1-h$ ) direction at $T=1.4 \mathrm{~K}$. Identical vertical scales emphasize variation in lineshape in the vicinity of $h_{\mathrm{c}}$. a-c, Solid lines, fits to single resonant mode (yellow shaded region) plus a higher energy continuum excitation (blue shaded region) convolved with the instrumental resolution function. For wavevectors $h \geq 0.2$, higher energy excitations are well represented by a two-particle continuum of the form $I=A \frac{\theta\left(\hbar \omega-\varepsilon_{1}(Q)\right) \theta\left(\varepsilon_{2}(Q)-\hbar \omega\right)}{\sqrt{(\hbar \omega)^{2}-\varepsilon_{1}^{2}(Q)}}$ where $\theta$ is a Heaviside step function and $\varepsilon_{2}(Q)$ is defined by the calculated upper boundary of the two-particle continuum (white line in Fig. 2a); $\varepsilon_{1}(Q)$ and $A$ were refined by the least squares fitting. d-f, For $h \leq 0.15$ this description fails and the spectrum is fitted by two superimposed damped harmonic oscillator spectra, each in the form of a difference of two lorentzians whose HWHM $\Gamma$ parameterizes damping, $I=\frac{\Gamma}{\pi}\left(\frac{1}{\Gamma^{2}+\left(\hbar \omega-\hbar \omega_{0}\right)^{2}}-\frac{1}{\Gamma^{2}+\left(\hbar \omega+\hbar \omega_{0}\right)^{2}}\right)$ (green shaded regions). The gaussian representing elastic incoherent nuclear scattering is also included at all wavevectors. Dashed lines and solid symbols in a-c show data on a one-fifth intensity $(I)$ scale. Error bars show statistical uncertainty estimated as the square root of total neutron count measured at each point. 
$h_{\mathrm{c}} \approx 0.15$ near the magnetic Brillouin zone boundary. The first frequency moment ${ }^{23}$ integrated over different ranges of energy transfer shown in Fig. 2b reveals how oscillator strength is transferred from the quasiparticle excitation to the multiparticle continuum, in analogy to what is observed in ${ }^{4} \mathrm{He}$ (ref. 13).

A change in the character of the excitation spectrum near $h_{\mathrm{c}}$ is also apparent in Fig. 3, which shows the energy-dependent magnetic scattering for wavevectors along the $(h, 0,-1-h)$ direction at $T \approx 1.4 \mathrm{~K} \ll \Delta_{s}$. For $h \geq 0.2$, Fig. 3a-c, there are two distinct contributions, a resolution-limited quasiparticle peak at lower energy and a broad feature with a sharp onset at higher energy, which we associate with the two-particle continuum. This continuum is well described by a square-root singularity above an energy threshold, typical for two-particle scattering governed by a diverging spectral density ${ }^{19}$. The threshold obtained from such data analysis is slightly higher than the calculated lower boundary of the two-magnon continuum (white line in Fig. 2a), and is close to the lowest energy of two-particle states involving gap mode magnons with a diverging DOS. Alternatively, the shift could indicate magnon repulsion.

For $h \leq 0.15$, the quasiparticle peak joins the continuum to form a complex spectral feature that extends from 2.5 to $4.5 \mathrm{meV}$ (Fig. 3d-f). We parameterize this spectrum by the overlapping response of two damped harmonic oscillators. The onset of scattering occurs well above the lowest energy for two non-interacting magnons (dashed white line in Fig. 2a), which indicates significant interactions. While the lower energy peak that appears to continue the quasiparticle dispersion in PHCC carries more spectral weight than the corresponding resonance at $Q>Q_{c}$ in superfluid ${ }^{4} \mathrm{He}$, it also has a measurable energy width as quantified in Fig. 2c. This demonstrates that a decay mechanism abruptly becomes accessible to the low energy excitations for $h \leq 0.15$. The width increases towards the Brillouin zone boundary, $h=0$, where the peak at the leading edge can be described by a non-quasiparticle square root singularity as used for the continuum at $h \geq 0.2$, or as an unstable non-dispersive resonance below the continuum.

The temperature dependence of scattering in ${ }^{4} \mathrm{He}$ for $Q$ between 2.4 and $2.6 \AA^{-1}$, Fig. 1 inset ${ }^{13,14}$, provides additional evidence of quasiparticle spectrum breakdown. Data in PHCC for $\mathbf{Q}=(0.15,0$, -1.15 ) where the one and two magnon states converge (Fig. $4 \mathrm{e}-\mathrm{h}$ ) similarly indicate that proximity to the two-particle continuum enhances thermal damping: the peak whose energy is approximately $20 \mathrm{~K}$ is already severely broadened at $T=10 \mathrm{~K}$ (Fig. $4 \mathrm{f}$ ). Its thermal broadening resembles that of the $\mathbf{Q}=(0.5,0,-1.5)$ gap mode, which is shown in Fig. 4a-d. This differs from observations in copper nitrate, a 1D QSL with weak dispersion where the one-magnon band lies well below the two-magnon continuum and decays cannot occur $^{24}$. Temperature-induced damping in that case is stronger for the lower-energy gap mode than for quasiparticles at the top of the dispersion curve; that is, heating mainly affects energy levels that become thermally populated. For PHCC, damping near the top and bottom of the band is governed by the same thermal population (Fig. 4a inset), consistent with the idea that high-energy excitations decay into gap-mode quasiparticles. As their thermal population increases, the probability of stimulated emission by the high-energy excitations also grows.

In summary, quasiparticle spectrum termination as seen in superfluid ${ }^{4} \mathrm{He}$ can also occur in other condensed matter systems, and in quantum magnets in particular. The dramatic changes observed in the spectrum of magnetic excitations in PHCC provide compelling evidence for such a phenomenon in the 2D QSL. The termination point is marked by rapid transfer of intensity from the magnon peak to the continuum at higher energies and by an abrupt appearance of damping. Although in PHCC the damped peak at the leading edge of magnetic scattering carries more intensity than the analogous peak in superfluid ${ }^{4} \mathrm{He}$, the lineshape and temperature dependence of postthreshold excitations in these two very different quantum liquids are remarkably similar.
Quasiparticles are ubiquitous in nature, ranging from phonons, magnons, rotons ${ }^{1-3}$, magnetorotons $s^{25}$ and heavy electrons and holes in condensed matter physics to quasiparticles of the quark gluon plasma and various unstable particles and resonances in the standard model of particle physics ${ }^{26}$. Rarely, however, do experiments offer as detailed a view of quasiparticle decay as the present results in a $2 \mathrm{D}$ organometallic spin liquid. Our findings show that an analysis of excitations in terms of quasiparticles with a welldefined dispersion relation can fail beyond a certain energymomentum threshold where the quasiparticles break down. This has important implications for a variety of condensed matter systems, in particular for other QSLs such as lamellar copper oxide superconductors, where spin excitations above a gap are considered as possible mediators of electron pairing and high-temperature superconductivity ${ }^{27}$.

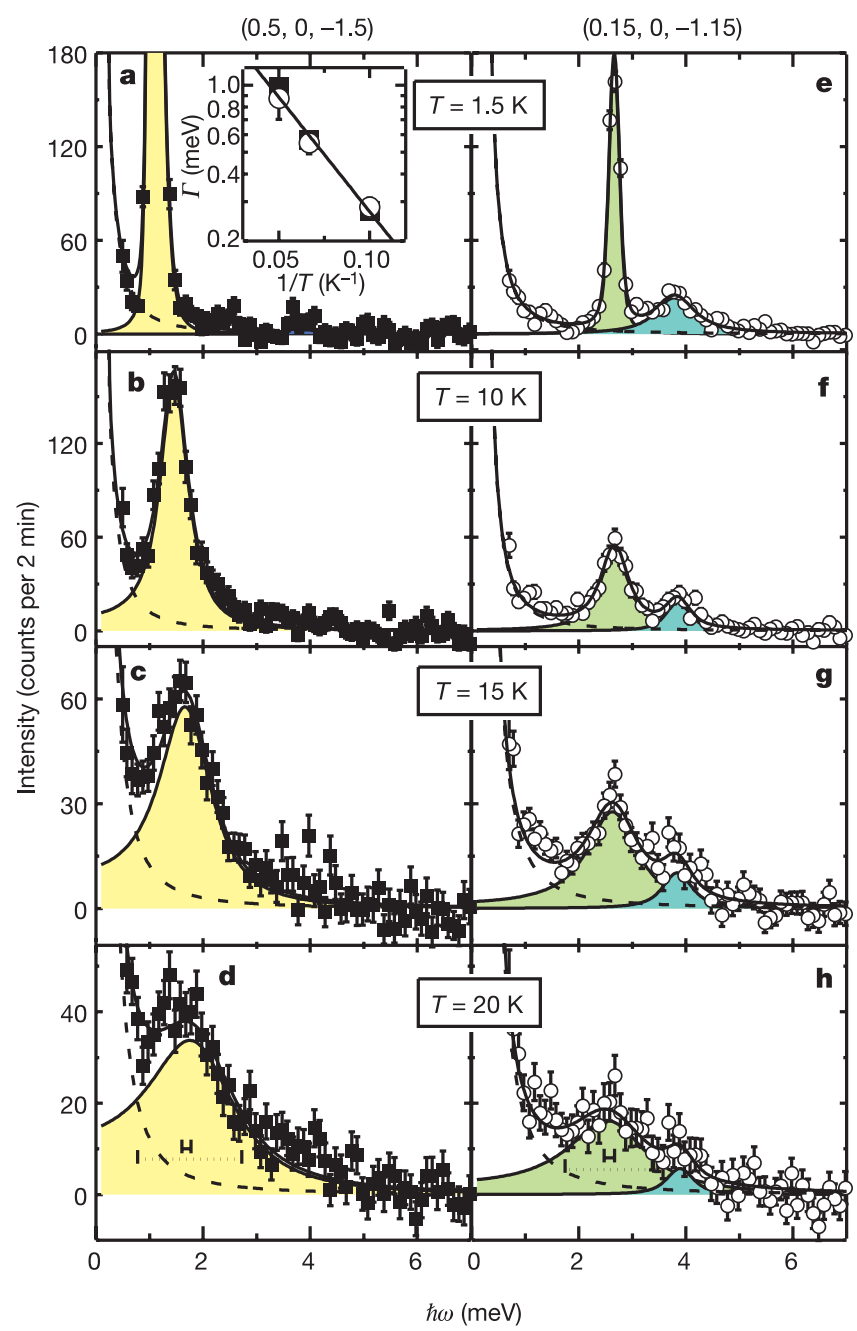

Figure 4 | Temperature dependent energy spectra for PHCC. Data are shown at $\mathbf{Q}=(0.5,0,-1.5)(\mathbf{a}-\mathbf{d})$ and $\mathbf{Q}=(0.15,0,-1.15)(\mathbf{e}-\mathbf{h})$. Solid lines for $T=1.5 \mathrm{~K}$ in $\mathbf{a}$ and $\mathbf{b}$ are fits as described in Fig. 3. Solid lines for $T \geq 10 \mathrm{~K}$ are fits to the following response function satisfying a detailed balance constraint: $S(Q, \omega)=\frac{\Gamma}{\pi}\left(\frac{1}{\Gamma^{2}+\left(\hbar \omega-\hbar \omega_{0}\right)^{2}}-\frac{1}{\Gamma^{2}+\left(\hbar \omega+\hbar \omega_{0}\right)^{2}}\right) \frac{1}{1-\exp (-\beta \hbar \omega)}$. The temperature dependence of the relaxation rate, $\Gamma$, for the lower energy peak at both wavevectors is shown in the inset to a. The line corresponds to exponentially activated behaviour with $\Delta=2.0 \mathrm{meV}$. Coloured areas below peaks indicate the assignment of different contributions to the spectra. Dashed lines indicate incoherent elastic nuclear scattering. Solid (dashed) horizontal bars in $\mathbf{d}$ and $\mathbf{h}$ indicate resolution (width of the low energy peak). Error bars show statistical uncertainty estimated as the square root of total neutron count measured at each point. 


\section{METHODS}

Neutron scattering measurements of $\left(\mathrm{C}_{4} \mathrm{H}_{12} \mathrm{~N}_{2}\right) \mathrm{Cu}_{2} \mathrm{Cl}_{6}$ (PHCC) were performed using the SPINS cold neutron triple axis spectrometer at the NIST Center for Neutron Research. Four deuterated PHCC crystals $^{5}$ with a total mass of $7.5 \mathrm{~g}$ were co-aligned to within $1^{\circ}$. Energy scans were acquired by varying the incident beam energy for fixed monitor counts in a low-efficiency detector between the pyrolytic graphite (PG (002)) monochromator and the sample. A $138^{\prime}$ radial collimator was used between the sample and a horizontally focusing PG (002) analyser with an angular acceptance of $5^{\circ}$ horizontally and $6^{\circ}$ vertically, which was followed by a matching single-channel, high-efficiency cylindrical detector. A cooled Be filter was placed after the sample. Measurements in Fig. 4 employed an additional PG filter before the sample. Data in Figs 2 and 3 were acquired with $5 \mathrm{meV}$ fixed final energy, and data in Fig. 4 at $3.7 \mathrm{meV}$ fixed final energy. Projected full-width at half-maximum energy resolution of these configurations at $\hbar \omega=0$ is $0.18 \mathrm{meV}$ and $0.11 \mathrm{meV}$, respectively. A wavevector independent fast-neutron background was measured by shielding the analyser entrance with cadmium. A wavevector-dependent thermal neutron background arising predominantly from incoherent phonon scattering was measured at $T=100 \mathrm{~K}$ and scaled using the thermal detailed balance factor for use as a lowtemperature non-magnetic background. These backgrounds were subtracted from all data presented.

\section{Received 12 August 2005; accepted 10 January 2006.}

1. Landau, L. D. \& Lifshitz, E. M. (eds) Course of Theoretical Physics Vol. 9, Statistical Physics Part 2 (by Lifshitz, E. M. \& Pitaevskii, L. P.) 125-139 (Pergamon, Oxford, 1981).

2. Abrikosov, A. A., Gorkov, L. P. \& Dzyaloshinski, I. E. Methods of Quantum Field Theory in Statistical Physics 235-247 (Dover Publications, New York, 1975).

3. Pitaevskii, L. P. Properties of the spectrum of elementary excitations near the disintegration threshold of the excitations. Sov. Phys. JETP 9, 830-837 (1959).

4. Kapitza, P. Viscosity of liquid helium below the $\lambda$-point. Nature 141,74 (1938).

5. Stone, M. B., Zaliznyak, I., Reich, D. H. \& Broholm, C. Frustration-induced two dimensional quantum disordered phase in piperazinium hexachlorodicuprate. Phys. Rev. B 64, 144405 (2001).

6. Anderson, P. W. The resonating valence bond state in $\mathrm{La}_{2} \mathrm{CuO}_{4}$ and superconductivity. Science 235, 1196-1198 (1987).

7. Xu, G. et al. Holes in a quantum spin liquid. Science $289,419-422$ (2000)

8. Rüegg, Ch. et al. Bose-Einstein condensation of the triplet states in the magnetic insulator $\mathrm{TICuCl}_{3}$. Nature 423, 62-65 (2003).

9. Stone, M. B. et al. Frustrated three-dimensional quantum spin liquid in $\mathrm{CuHpCl}$. Phys. Rev. B 65, 064423 (2002).

10. Gopalan, S., Rice, T. M. \& Sigrist, M. Spin ladders with spin gaps: A description of a class of cuprates. Phys. Rev. B 49, 8901-8910 (1994).

11. Woods, A. D. B. \& Cowley, R. A. Structure and excitations of liquid helium. Rep. Prog. Phys. 36, 1135-1231 (1973).
12. Smith, A. J., Cowley, R. A., Woods, A. D. B., Stirling, W. G. \& Martel, P. Roton-roton interactions and excitations in superfluid helium at large wavevectors. J. Phys. C 10, 543-553 (1977).

13. Fåk, B. \& Bossy, J. Temperature dependence of $S(Q, E)$ in liquid ${ }^{4} \mathrm{He}$ beyond the roton. J. Low Temp. Phys. 112, 1-18 (1998).

14. Montfrooij, W. \& Svensson, E. C. Superfluid ${ }^{4}$ He: just a very cold liquid? J. Low Temp. Phys. 121, 293-302 (2000).

15. Pistolesi, F. Theory and data analysis for excitations in liquid ${ }^{4} \mathrm{He}$ beyond the roton minimum. Phys. Rev. Lett. 81, 397-400 (1998).

16. Ruvalds, J. \& Zawadowski, A. Theory of structure in the superfluid helium spectrum considering roton-roton resonances. Phys. Rev. Lett. 25, 333-337 (1970).

17. Feynman, R. P. \& Cohen, M. Energy spectrum of the excitations in liquid helium. Phys. Rev. 102, 1189-1204 (1956).

18. Giamarchi, T. Quantum Physics in One Dimension (International Series of Monographs on Physics, Oxford Univ. Press, Oxford, UK, 2005).

19. Meshkov, S. V. Monte-Carlo study of quantum spin chains. Phys. Rev. B 48 6167-6178 (1993).

20. Regnault, L.-P., Zaliznyak, I. A. \& Meshkov, S. V. Thermodynamic properties of the Haldane spin chain: statistical model for the elementary excitations. J. Phys. Condens. Matter 5, L677-L683 (1993).

21. Ma, S., Broholm, C., Reich, D. H., Sternlieb, B. J. \& Erwin, R. W. Dominance of long-lived excitations in the antiferromagnetic spin-1 chain NENP. Phys. Rev. Lett. 69, 3571-3574 (1992).

22. Zaliznyak, I. A., Lee, S.-H. \& Petrov, S. V. Continuum in the spin-excitation spectrum of a Haldane chain observed by neutron scattering in $\mathrm{CsNiCl}_{3}$. Phys. Rev. Lett. 87, 017202 (2001)

23. Hohenberg, P. C. \& Brinkmann, W. F. Sum rules for the frequency spectrum of linear magnetic chains. Phys. Rev. B 10, 128-131 (1974).

24. Xu, G., Broholm, C., Reich, D. H. \& Adams, M. A. Triplet waves in a quantum spin liquid. Phys. Rev. Lett. 84, 4465-4468 (2000).

25. Girvin, S. M., MacDonald, A. H. \& Platzman, P. M. Magneto-roton theory of collective excitations in the fractional quantum Hall-effect. Phys. Rev. $B$ 33, 2481-2494 (1986)

26. Cottingham, W. N. \& Greenwood, D. A. An Introduction to the Standard Model of Particle Physics (Cambridge Univ. Press, Cambridge, UK, 1998).

27. Tranquada, J. M. et al. Quantum magnetic excitations from stripes in copper oxide superconductors. Nature 429, 534-538 (2004)

Acknowledgements We acknowledge discussions with L. Passell, J. Tranquada, A. Abanov, M. Zhitomirsky, A. Tsvelik, A. Chitov and M. Swartz. Work at BNL and ORNL was supported by the US Department of Energy. Work on SPINS and at JHU was supported by the US National Science Foundation. We are grateful to B. Fåk for permission to reproduce the ${ }^{4} \mathrm{He}$ results in Fig. 1 and to A. Zaliznyak for help with the manuscript.

Author Information Reprints and permissions information is available at npg.nature.com/reprintsandpermissions. The authors declare no competing financial interests. Correspondence and requests for materials should be addressed to I.A.Z. (zaliznyak@bnl.gov). 